\title{
Nano Porous Films Processing of Polymer Films Based on Localized Surface Plasmon Resonance of Au Nanoparticles
}

\author{
Keita MURAOKA ${ }^{1}$, Kazushi YAMADA $^{2}$, Tatsuya SHOJI ${ }^{1}$, \\ Hiroyuki SUGIMURA ${ }^{3}$, Noboru KITAMURA ${ }^{1}$ and Yasuyuki TSUBOI ${ }^{1,4^{*}}$
}

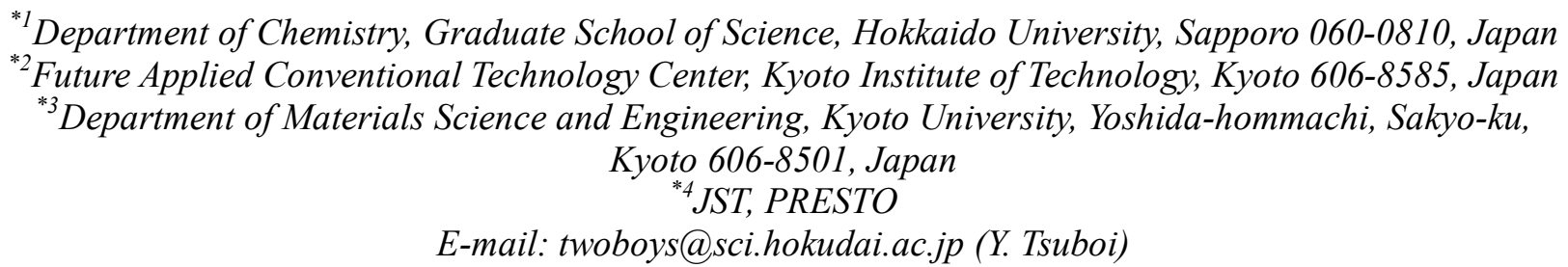

We present a novel laser processing technique for nanoholes formation $(d \sim 100 \mathrm{~nm})$ on a polymer film based on resonant excitation of surface plasmon of Au nanoparticles. We succeeded in fabrication of a porous film with controlling of the density and position of the nanoholes in the film. The fundamental mechanism of the processing is superheating of $\mathrm{Au}$ nanoparticles by resonant excitation, with subsequent explosive vaporization to leave nanoholes.

DOI:10.2961/jlmn.2012.03.0005

Keywords: Au nanoparticles ; self-assembled monolayer ; Laser Ablation ; Diffraction limit ; YAG laser

\section{Introduction}

Laser micro/nano processing technology for various materials has undergone explosive growth and development in the past two decades. For laser etching or laser hole processing, the spatial resolution of processing is regulated by a diffraction limit of light. In particular for nanosecond visible laser processing, it is impossible to create a nonahole whose diameter is smaller than $\sim 300 \mathrm{~nm}$. This is a crucial technical problem in laser micro/nano processing in general, since nanosecond pulsed lasers are nowadays widely spread in the world.

As a solution to create a nanohole with $d<300 \mathrm{~nm}$, recent trend in this research field falls into two categories. One of major approaches is to use ultrafast (ps-fs) pulsed lasers for processing transparent materials. Since multiphoton absorption is efficiently induced only in a central area of a Gaussian beam spot (where density of a photon flux is extremely high), ps-fs laser processing can create a nano-void or nanohole beyond the diffraction limit of laser light in a transparent material through multi-photon absorption. The other approach is to use a pulsed laser oscillating at the vacuum ultraviolet (VUV) wavelength region. The use of shorter laser wavelengths is favorable for more-precise, finer processing. These two approaches take advantages of the characteristics of laser light, and have been examined by numerous research groups.
Contrary to such approaches from laser sources, we recently proposed a novel alternative technique that was approached from a material side to form small nanoholes ( $d$ $\leqq 100 \mathrm{~nm}$ ) in a polymer film ${ }^{1)}$. By irradiation to $\mathrm{Au}$ nanoparticles embedded in a polymer film with visible laser light, it is possible to process a small nanohole overcoming the diffraction limit of laser light. The present technique bases on excitation of localized surface plasmon of $\mathrm{Au}$ nanoperticls. Au nanoparticles are superheated, immediately followed by explosive vaporization to leave nanoholes in the film. The diameter of these nanoholes was less than $100 \mathrm{~nm}$, which was much smaller than the wavelength of the laser used $532 \mathrm{~nm}$. The concept of this technique is schematically drawn in Fig.1.

In the previous report, we preliminary demonstrated such small nanoholes formation beyond the diffraction limit of laser light. However, detailed feature of the technique still remain unclear and improvements of the technique is further necessary. Indeed, we have not controlled the density and position of nanoholes in a polymer film. In the present study, we demonstrate the control of the number of a nanohole and fabrication of a nanoporous polymer film. 


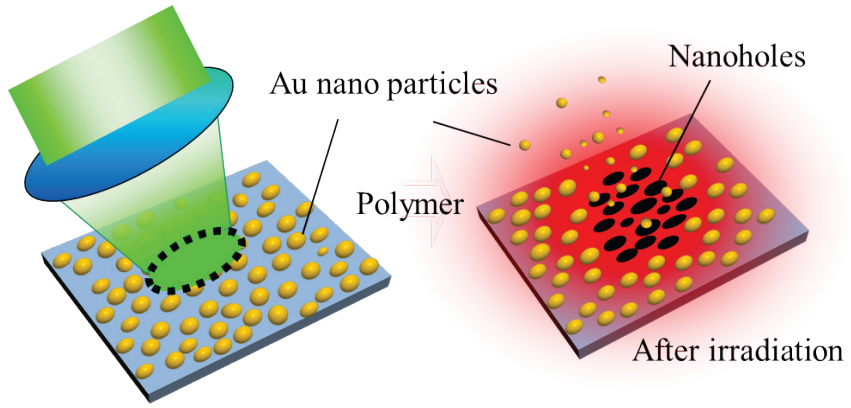

Fig. 1 Schematic illustrations of the microfabrication of our present technique with laser ablation.

\section{Experiment}

The technique is based on the previous report. We adopted a silane coupling method to prepare a selfassembled monolayer (SAM) on a glass surface ${ }^{2)}$. In the SAM, of (3-aminopropyl) trimethoxysilane (SigmaAldrich Co.) play a role as a linker. The SAM process is necessary in order to fix individual $\mathrm{Au}$ nanoparticles separately on a glass substrate, avoiding their aggregation. Then, we cast a colloidal solution of $\mathrm{Au}$ nanoparticles (British Biocell International Ltd., diameter; $d=30 \mathrm{~nm}$ ) on the glass surface with keeping high humidity in the vessel. Thus, a lot of $\mathrm{Au}$ nanoparticles were linked onto the glass substrate by SAM. After that, we spin-coated PMMA (average molecular weight $\sim 10^{5}$ ) onto the Au-nanoparticlefixed-glass substrate. The film thickness was adjusted to be approximately $20 \mathrm{~nm}$. The sample film thus prepared showed an absorption band around $530 \mathrm{~nm}$, which is due to the resonant transition of surface plasmon of $\mathrm{Au}$ nanoparticles. The film samples were irradiated with the second harmonic output of a pulsed $\mathrm{Nd}^{3+}$ :YAG laser (532 $\mathrm{nm}, \mathrm{FWHM} \sim 10 \mathrm{~ns})$ in single-shot-mode with a fluence ranging from $400 \sim 800 \mathrm{~mJ} / \mathrm{cm}^{2}$. The laser light was linearly polarized. The surface morphology of the target substrates were analyzed using an atomic force microscope (AFM, Seiko Instruments Inc., SPI3800N, NanoNavi II) in Dynamic Force Microscope (DFM) mode. All of the experiments were carried out under an atmospheric condition.

\section{Result and Discussion}

\subsection{Density control of Au nanoparticle integrated on a glass substrate}

As a representative example, Fig. 3 shows AFM images of film surfaces prepared by the manner described above. Since the diameter of $\mathrm{Au}$ nanoparticles $(d=30 \mathrm{~nm})$ was larger than the film thickness $(20 \mathrm{~nm})$, a top part of $\mathrm{Au}$ nanoparticles are recognized on a sample polymer film. The density of $\mathrm{Au}$ nanoparticles in Fig. 2 was evaluated to be $50 \mu^{-2}$. By changing the experimental condition (i.e., immersion time of the Au colloidal solution to the SAM substrates) in the procedure of film preparation, we succeeded in increasing the number of Au nanoparticles embedded in a polymer film up to $150 \mu^{-2}$, as shown in Fig. 3 .Thus, we were able to control the density of $\mathrm{Au}$ nanoparticles integrated on the SAM substrates by changing the immersion time. These substrates were used to fabricate nanoporous films.



Fig. 2 AFM images of target sample films before



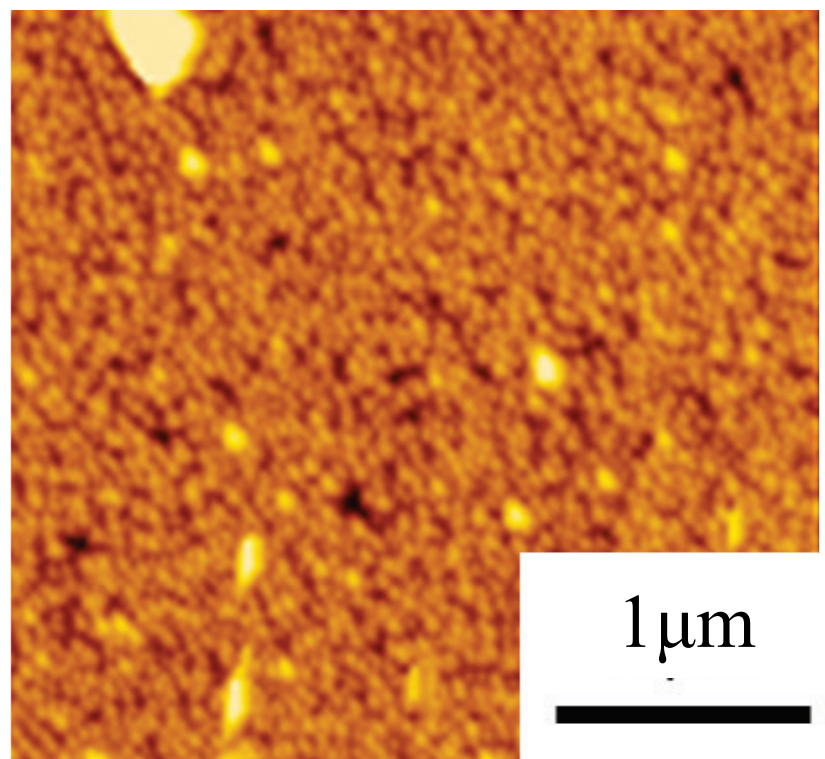

Fig. 3 AFM images of target sample films before irradiation (high density; $150 \mu \mathrm{m}^{-2}$.)

\subsection{Fabrication of nanoporous films}

Fig. 4 shows a representative AFM image of a sample film surface after laser irradiation at $400 \mathrm{~mJ} / \mathrm{cm}^{2}$, using a target film of Fig.3. Black area corresponds to processed nanoholes in the image, whereas white points correspond to $\mathrm{Au}$ nanoparticles that were not evaporated. We estimated the density of nanoholes to be approximately $100 \mu^{-2}$. Considering the number of density of $\mathrm{Au}$ nanoparticles fixed to the glass substrate before laser irradiation (150 $\mu \mathrm{m}^{-2}$ ), about $65 \%$ of Au nanoparticles were converted into nanoholes by single-shot laser irradiation. Although the laser fluence at $400 \mathrm{~mJ} / \mathrm{cm}^{2}$ was sufficient to form a nano porous film, increasing laser intensity enable us form smaller and numerous nanoholes. 
In other words, the density of nanoholes was increased by increasing the laser intensity. The density of nanoholes was estimated to be approximately $130 \mu^{-2}$, at $600 \mathrm{~mJ} / \mathrm{cm}^{2}$, indicating that about $85 \%$ of Au nanoparticles were converted to nanoholes. From the AFM image, the average size $(d)$ of nanoholes was estimated to be ca. 70 $\mathrm{nm}$ at $400 \mathrm{~mJ} / \mathrm{cm}^{2}$. It was shown that, the diameter of nanoholes was close to that of $\mathrm{Au}$ nanoparticles $(d \sim 30$ $\mathrm{nm})$. Fig. 5 shows an AFM image of a nano porous film formed by laser irradiation at $600 \mathrm{~mJ} / \mathrm{cm}^{2}$. The average size (d) of nanoholes was estimated to be ca. $50 \mathrm{~nm}$ at 600 $\mathrm{mJ} / \mathrm{cm}^{2}$. It should be noted that the diameter of nanoholes decreased in comparison with that formed by laser irradiation at $400 \mathrm{~mJ} / \mathrm{cm}^{2}$ (Fig.4). In this case,

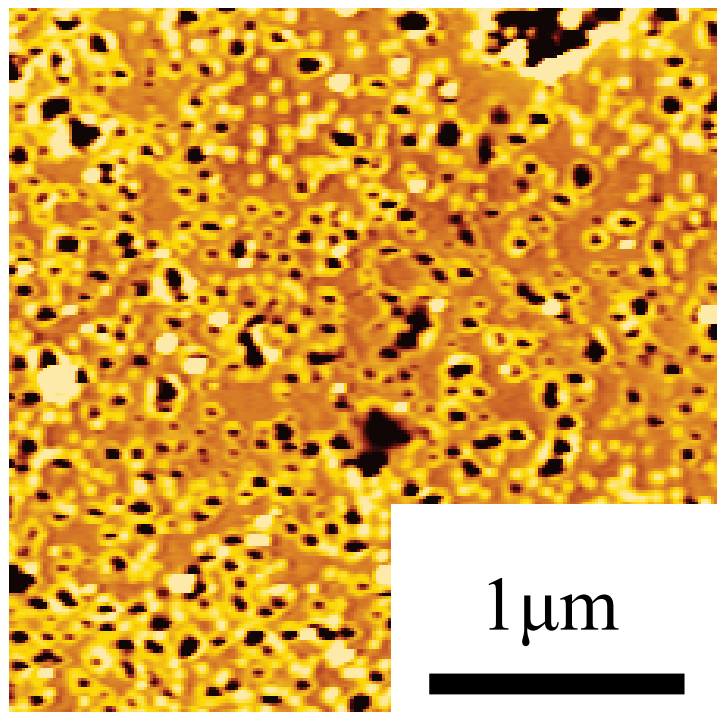

Fig. 4 AFM image of the surface of the substrate after laser irradiation (single shot of a 532-nm laser pulse at 400 $\mathrm{mJ} / \mathrm{cm}^{2}$ ).

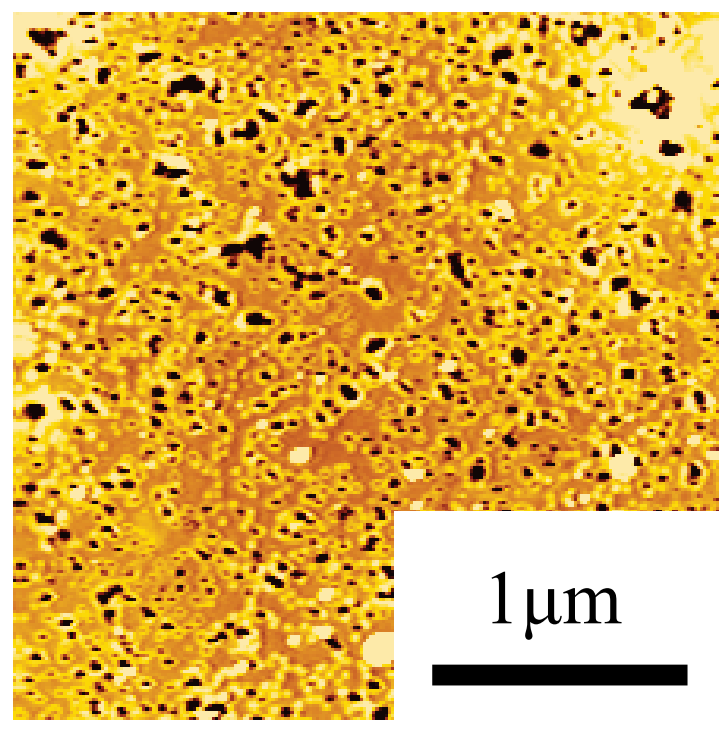

Fig. 5 AFM image of the surface of the substrate after laser irradiation (single shot of a 532-nm laser pulse at 600 $\mathrm{mJ} / \mathrm{cm}^{2}$ ).

\subsection{Patterning of nanoholes}

The patterned nanohole formations were conducted by using stripe-patterned SAM substrates. The substrates were prepared by the VUV light (172 nm) lithography under nitrogen atomosphere. ${ }^{3)}$ Fig.6 shows the AFM images and the schematic illustrations of the substrates before and after laser irradiation, respectively. Before irradiation, the bulges with the average height of $20 \mathrm{~nm}$ were observed like stripe shape. This can be readily explained by the selective adsorption of $\mathrm{Au}$ nanoparticles on the substrate. The nanoholes with the diameter and depth of 30 and $20 \mathrm{~nm}$, respectively, were observed after laser irradiation. The nanoholes aligned along the stripeshape pattern as well as bulges before irradiation. These results strongly suggest that the nanoholes were formed by the existence of the Au nanoparticles. Therefore the stripe patterned nanoholes can be formed by using stripe patterned SAM substrates. Although spatial resolution and contrast were not clear since this was a primary demonstration, the technique would be potentially applicable to form precise micro-pattern formation with nanoholes.

(a)
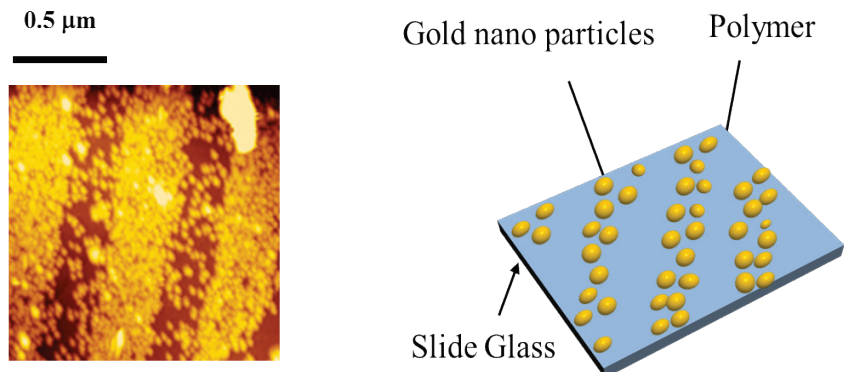

(b)

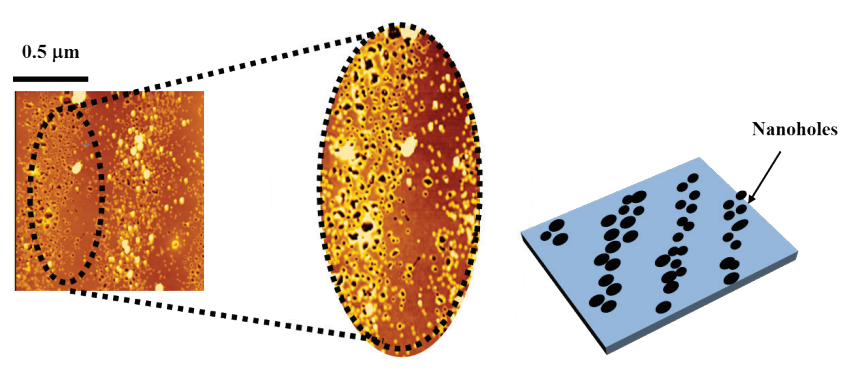

Fig. 6 AFM image and schematic illustrations of the stripepatterned $\mathrm{Au}$ substrate after laser irradiation (a) and after irradiation (b). 


\subsection{Processing mechanisms}

The depth of nanoholes was revealed to be much close to the thickness of the film (ca. $20 \mathrm{~nm}$ ), indicating that nanoholes completely penetrated the sample polymer film. The depths of the nanoholes formed at the both laser intensity were almost the same as the film thickness. However in Fig. 4 and Fig.5, the diameters of the nanoholes were somewhat larger than that of the $\mathrm{Au}$ nanoparticles used here. The change in size can be explained in terms of two factors. The first factor is presumably ascribable to the explosive vaporization (vapor jet along the lateral direction) and to thermal diffusion in the lateral direction. Indeed, the thermal diffusion length $l_{d \mathrm{t}}$ is given by

$l_{d t}=(\kappa \tau)^{1 / 2}$

where $\kappa$ is thermal diffusion coefficient of PMMA and $\tau$ is the pulse duration of the incident laser. Equation (1) gives $l_{d t} \leqq 100 \mathrm{~nm}$, which is consistent with the AFM observation. Thus the increase in the size of nanoholes is probably due to thermal diffusion. The second factor is coalescence of nanoholes, which should be appreciable with increasing the density of nanoholes.

In addition, the measured and calculated values of the diameter were very close. In this way, we can control the size and density of nanoholes simply by varying laser intensity. Thus, we safely conclude that the present method can create numerous nanoholes with the diameter overcoming diffraction limit of irradiation laser light.

In order to fabricate smaller nanoholes with $d \leq 100$ $\mathrm{nm}$, we had better utilize surface plasmon at a "nanogap" between adjacent metallic nanostructures (gap-mode plasmon). Gap-mode plasmon accelerates photochemical reaction due to enhancement of incident electromagnetic field $^{4)}$. Recently, such naogap-based localized plasmon was applied for nano lithography ${ }^{5}$. This technique enables us to overcome the diffraction limit of light. Such gapmode surface plasmon is also applicable to our technique and such an approach is currently in progress.

\section{Summary}

We have demonstrated a novel sub-wavelength laser processing technique. Nanoholes with the diameter of several tens-of-nanometers were formed in the polymer film by resonant excitation of Au nanoparticles. The origin of the nanohole formation would be explosive vaporization of the Au nanoparticles via superheated state induced by nanosecond $532 \mathrm{~nm}$ pulsed laser irradiation. We are able to control the density and position of small nanoholes in a sample polymer film. Such polymer films containing numerous nanoholes would have potential applications in the fields of biomaterials and electronic devices. It should be noted that $\mathrm{Au}$ nanoparticles-based-nanoholes-processing is applicable to a glass processing demonstrated by Hashimoto's ${ }^{6,7,8,)}$ and Miyanishi's groups ${ }^{9)}$.

\section{Acknowledgments}

This work was partly supported by a Grant-in-Aid for Scientific Research from the Ministry of Education, Culture, Sports, Science and Technology of Japan to the Priority Area "Strong Photon-Molecule Coupling Fields (470)" (No. 19049004), No. 20550002, and the Global COE Program (Project No. B01: Catalysis as the Basis for Innovation in Materials Science).

\section{References}

[1] K. Yamada, T. Itoh, Y. Tsuboi, Appl. Phys. Express, 1, No. 8 (2008) 087001

[2] Y. Tsuboi, R. Shimizu, T. Shoji, J. Am. Chem. Soc. 131, (2009) 12623-12627

[3] N. Saito, Y. Wu, K. Hayashi, H. Sugimura and O. Takai, J. Phys. Chem. B. 107 (2003) 664

[4] Y. Tsuboi, T. Shoji, N. Kitamura, M. Takase, K. Murakoshi, Y. Mizumoto, H. Ishihara, J. Phys. Chem. Lett., 1 (2010) 2327-2333

[5] K. Ueno, S. Takabatake, Y. Nishijima, V. Mizeikis, Y. Yokota, and H. Misawa, J. Phys. Chem. Lett., 1, 3, (2010) 657-662

[6] S. Hashimoto, T. Uwada, M. Hagiri, J. Phys. Chem. C, 115, (2011) 4986-4993.

[7] D. Werner, S. Hashimoto, T. Uwada: Langmuir, 12, (2010) 9956-9963

[8] S. Hashimoto, T. Uwada, M. Hagiri. J. Phys. Chem. C, 113, (2009) 20640-20647

[9] T. Miyanishi, T. Sakai, N. Nedyalkov. N, Appl. Phys. A-Materials Science \& Processing, 96 (2009) 843-850

(Received: October 04, 2011, Accepted: July 05, 2012) 